\title{
Barotropic Instability of an Easterly Zonal Current*
}

\author{
By Masanori Yamasaki** and Misuzu Wada \\ New York University, Meteorological Research Institute, Tokyo \\ (Manuscript received 3 August 1971, in revised form 10 December 1971)
}

\begin{abstract}
As an extension of Nitta and Yanai (1969) the stability properties of a barotropic easterly current with a sine-profile jet are studied in more detail. The barotropic vorticity equation on a $\beta$-plane is solved by the finite difference method, as was made in the previous study. It is found that the stability properties of the easterly current are different from those of the westerly current in several respects such as a possibility of the existence of an unstable wave which propagates faster than a maximum velocity of an easterly current, the existence of many unstable waves of various meridional modes, strong dependency of wave properties on lateral boundaries, latitudinal profiles of wave amplitudes (as well as the direction of wave axis) and so on. It is also found that the $\beta$-plane approximation is justified only when we discuss two unstable waves of the lowest and second meridional modes.
\end{abstract}

\section{Introduction}

The wave disturbances observed in the trade easterlies in the tropical troposphere are known as "easterly waves." The energy source of the easterly wave has been a controversial problem for many years. So far three possibilities have been proposed. The first possibility is the energy propagation from the middle latitudes (Mak, 1969; Charney, 1969; Nitta, 1970 a). However, this possibility does not appear to be very significant to the easterly wave when we admit Mak (1969)'s result that the longitudinal wavelength of a wave which is most excited by this mechanism is more than several thousand kilometers. As is well known, the scale of the easterly wave is usually 1,500 6,000 km (Riehl, 1954; Palmer, 1952; Wallace and Chang, 1969; Chang, 1970; Chang et al., 1970; Yanai et al., 1968 and others). The second possibility is the available potential energy generated by condensation heat released by convective clouds in the conditionally unstable tropics (Manabe and Smagorinsky, 1967; Manabe et al., 1970; Krishnamurti, 1969; Yamasaki, 1969,

* Contribution No. 114, Geophysical Sciences Laboratory, Dept. of Meteorology and Oceanography, New York University. This research has been partially supported by National Science Foundation under Grant GA-12568.

** Permanent affiliation: Meteorological Research Institute, Tokyo.
1971; Hayashi, 1970; Ooyama, 1971; Nitta, 1970b; Chang, et al., 1970 and others). The third is the barotropic instability associated with the horizontal shear of the easterlies. In view of the observational fact that the easterly wave usually has a cold-cored structure in the lower troposphere, some authors (Yanai, 1961 and others) suggested this possibility as one of possible mechanisms for the growth of the easterly wave. Recently this problem was discussed by Nitta and Yanai (1969) and Lipps (1970). The present authors are interested in the properties of a wave which is caused by combined effects of the second and third possibilities. This paper is a preliminary report in which only the third problem is discussed as the authors' first step for that purpose.

The stability property of a barotropic zonal current with a "westerly" jet has been fairly clarified by many authors (Kuo, 1949; Eliasen, 1954; Wiin-Nielsen, 1961; Haltiner and Song, 1962; Lipps, 1962, 1965; Yanai and Nitta, 1968). The results by these studies, however, cannot be directly applied to the problem of stability of an "easterly" jet because of the effect of the earth's rotation. According to Nitta and Yanai (1969) and Brown (1969), as far as an easterly jet with a sine-profile is concerned, all waves longer than a certain wavelength are unstable if a necessary condition for instability (Kuo, 1949) is satisfied. In the authors' linear analysis of a three-dimensional quasi-geostrophic model which 
was made preceeding the present study, the authors noticed that there exists an upper critical wavelength for instability under some conditions and that the unstable waves do not necessarily propagate westward slower than a maximum easterly current. These results motivated the authors to re-examine the stability property of an easterly jet by the use of a two-dimensional model.

\section{Stability of a sine-profile easterly jet bounded by two walls}

The model considered in this section is the same as that used by Nitta and Yanai (1969); that is, our problem is to solve the linearized barotropic vorticity equation

$$
(U-c)\left(-k^{2} \phi+\phi^{\prime \prime}\right)+\left(\beta-U^{\prime \prime}\right) \psi=0
$$

under the boundary conditions

$$
\phi=0 \quad \text { at } y= \pm b
$$

for a given profile of the basic zonal flow

$$
U=-U_{\max } \cos ^{2} \frac{\pi}{2 b} y
$$

The notations are $\phi=$ amplitude of the perturbation streamfunction (complex in general), $y=$ north-south coordinate whose origin is taken so as to coincide with the center of a jet, $k=2 \pi / L$, $L=$ longitudinal wavelength of a wave, $U_{\max }=$ maximum intensity of an easterly current, $2 b=$ width of the belt under consideration, $\beta=d f / d y$, $f=$ the Coriolis parameter and prime denotes a differentiation with respect to $y$. When we write $c=c_{r}+i c_{i}, c_{r}$ is the phase velocity in the eastward direction and $q=k c_{i}$ is the growth rate.

We shall obtain eigenvalues $c$ and eigensolutions $\psi$ by the finite difference method. The validity of this method in the present problem was discussed by Yanai and Nitta (1968). The scheme of the finite difference used is also the same as used by Nitta and Yanai (1969) and Yanai and Nitta (1968). When we divide the belt under consideration into $2 N$ subdivisions and apply equation (2.1) at interior grids, we have $N$ symmetric waves and $(N-1)$ antisymmetric waves with respect to $y=0$. Since antisymmetric waves do not become unstable in the present model with the boundary condition (2.2) (Nitta and Yanai, 1969), we shall limit our

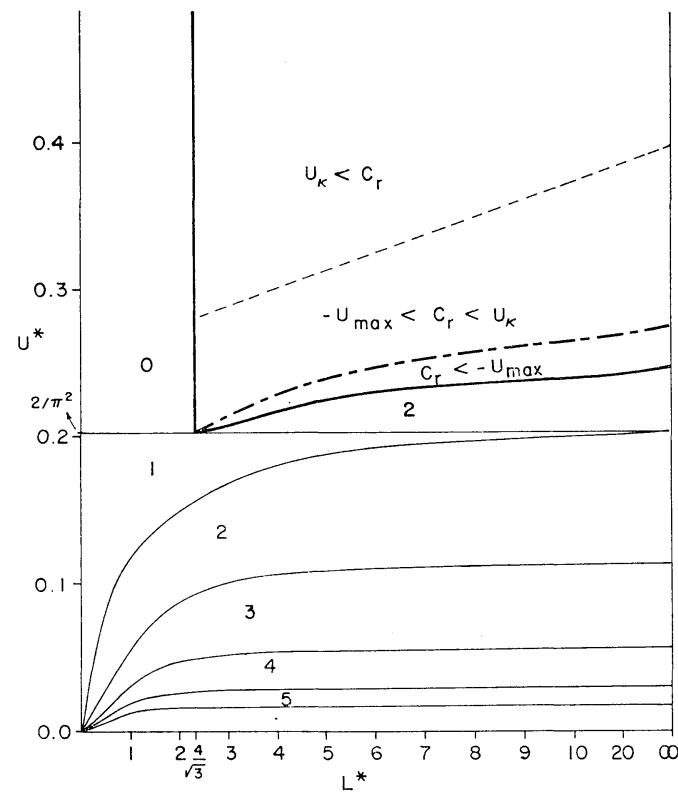

Fig. 1. The condition for the occurrence of an unstable wave. Lower critical values of non-dimensional wavelength $L^{*}(=L / b)$ and lower critical values of $U^{*}\left(=U_{\max } / \beta b^{2}\right)$ are indicated by the thick solid lines. The regime for the unstable wave is divided into three domains by its phase velocity. The numerals indicate the number of neutral waves (regular solutions) of symmetric mode.

discussions to symmetric waves.

The stability property of the zonal current (2.3) is determined by two dimensionless parameters $U^{*}=U_{\max } / \beta b^{2}$ and $L^{*}=L / b$ (Lipps, 1962; Yanai and Nitta, 1968). The parameter $U^{*}$ is a measure of the horizontal shear of the easterly current as well as the effect of the earth's rotation. Fig. 1 shows the property of waves with $L^{*}$ as the abscissa and $U^{*}$ as the ordinate. This diagram was obtained for $2 N=20$.

For $U^{*}<2 / \pi^{2} \fallingdotseq 0.203$, the quantity $Z$ has a positive sign throughout the domain, where $Z$ is the meridional gradient of the absolute vorticity of the basic zonal current

$$
Z=\beta-U^{\prime \prime} \text {. }
$$

In this case, as shown by Kuo (1949), we do not obtain any unstable waves but have several modes of Rossby-Haurwitz waves which move westward faster than a maximum velocity of the 



Fig. 2. Phase velocity diagrams for various values of $U^{*}$. The quantity $c_{r} *$ is defined as $c_{r} / U_{\max }$. The thin solid lines indicate neutral waves (regular solutions) and the thick solid lines indicate unstable waves. The white circles indicate a neutral wave with $c_{r}=U \kappa$, and black circles (except for Fig. $2 \mathrm{k}$ ) and dashed lines are neutral waves which correspond to weak solutions of equation (2.1).

easterly current. The number of Rossby-Haurwitz waves depends upon $U^{*}$ and $L^{*}$. This is shown by numerals in Fig. 1. As for the case of a westerly current, Yanai and Nitta (1968) obtained this kind of diagram (including antisymmetric mode).

The phase velocity diagrams obtained for $U^{*}$ $=0.180$ and 0.195 are shown in Figs. $2 \mathrm{a}$ and $2 \mathrm{~b}$ for some range of $c_{r}^{*}=c_{r} / U_{\max }$. The ordinate is $c_{r} *$ and the abscissa is $L^{*}$. Rossby-Haurwitz waves are shown by the solid lines. The dashed lines and black circles indicate the solutions of the finite difference equations which correspond to weak solutions of the differential equation.

For $U^{*}=2 / \pi^{2}, Z$ becomes zero at $y=0$. The phase velocity diagram for this case is shown in Fig. 2c. According to the theory by Kuo (1949) there exists a neutral wave with a phase velocity which is equal to $U$ at a latitude where $Z=0$. The wavelength of this neutral wave is given by $L=4 b / \sqrt{3}$ (Nitta and Yanai, 1969). This wave is indicated by the open circle in Fig. 2c. (This wavelength obtained by the finite difference method is slightly different from $4 b / \sqrt{3}$.) For 
$L^{*}<4 / \sqrt{3}$, there does not exist any RossbyHaurwitz wave. One Rossby-Haurwitz wave exists only for $L^{*}>4 / \sqrt{3}$. The solution indicated by the chain line (except for $L^{*}=4 / \sqrt{3}$ ) is not a solution of the differential equation (2.1). Such false solutions are obtained in the case where $Z$ vanishes at grid points.

When $U^{*}$ exceeds $2 / \pi^{2}$, barotropic unstable waves appear for $L^{*}>4 / \sqrt{3}$, as shown by Nitta and Yanai (1969). There is an upper limit to wavelength for instability provided that $U^{*}<0.246$. The lower and upper critical curves for instability are given by the thick solid lines in Fig. 1. The phase velocity diagrams for $U^{*}=0.210,0.213$ and 0.230 are shown in Figs. 2d, 2e and 2f, respectively. The thick solid lines indicate unstable waves. One of remarkable results is that for some range of wavelengths there is an unstable wave which moves faster than the zonal flow $\left(c_{r}<-U_{\max }\right)$. For larger wavelengths we have two kinds of neutral waves with $c_{r}<-U_{\max }$. When $U^{*}$ is larger than about 0.246 , neutral waves with $c_{r}<$ $-U_{\max }$ disappear (Fig. 2g). Then there is no upper limit to wavelengths for instability. For $U^{*}>0.27$ the phase velocity of all the unstable waves is larger than $-U_{\max }$ (Figs. $2 \mathrm{~h}, 2 \mathrm{i}, 2 \mathrm{j}$ ). The chain line in Fig. 1 indicates the boundary between a regime of an unstable wave with $c_{r}>-U_{\max }$ and that with $c_{r}<-U_{\max }$.

The regime for the occurrence of an unstable wave with $c_{r}>-U_{\max }$ can be divided into two regimes bounded by the dashed line in Fig. 1. For smaller values of $U^{*}$ the unstable wave moves westward faster than $U_{k}$, an easterly current at a latitude where $Z$ is zero. For larger values of $U^{*}$, the wave moves westward slower than given by $c_{r}=U_{k}$. The phase velocity diagrams for $U^{*}=0.28,0.33$ and 0.45 are shown in Figs. $2 \mathrm{~h}, 2 \mathrm{i}$ and $2 \mathrm{j}$, respectively. When $U^{*}$ is 0.33 , we find an unstable wave which propagates at a velocity of $U_{k}$ (for $L^{*} \fallingdotseq 6$ ).

The phase velocity diagram for $\beta=0$ ( $U^{*}$ is infinite) is shown in Fig. 2k. As seen from equation (2.1), for infinite $L^{*}, c$ is equal to $U$ at a latitude where the boundary condition $\phi=0$ is imposed. In fact, a wave with $c=0$ and $\phi=\alpha U$ ( $\alpha$ : a constant) exists for infinite $L^{*}$ (black circle in Fig. 2k). The solid line in Fig. 2k should be connected with the black circle if the number of sub-divisions used in the finite difference increases to infinity. Then there would be no upper limit

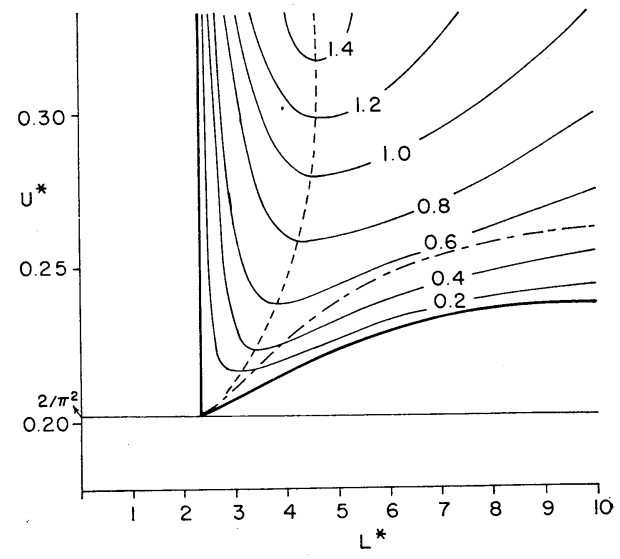

Fig. 3. Growth rate (unit: $\beta b / 20$ ) as a function of $U^{*}$ and $L^{*}$.

to wavelength for instability.

The phase velocity and wavelength of a neutral wave indicated by the open circle in Fig. 2c to Fig. 2k are slightly different from theoretical values. The normalized phase velocity of this neutral wave is theoretically given by

$$
c_{r}^{*}=-\left(1 / 2+1 / \pi^{2} U^{*}\right)
$$

and the wavelength is $4 / \sqrt{3} b$. The difference between theoretical values and those obtained by the finite difference method was discussed by Yanai and Nitta (1968).

Fig. 3 shows the growth rate of the unstable wave as a function of $U^{*}$ and $L^{*}$ (unit: $\beta b / 20$ ). It is seen that a wave with $c_{r}>-U_{\max }$ is the most preferrred mode for any values of $U^{*}$. It is also seen that the growth rate of the unstable wave with $c_{r}<-U_{\max }$ is smaller than $10^{-6} \mathrm{sec}^{-1}$ when we take $2 b \sim 2,000 \mathrm{~km}$.

The results described above differ from those of Nitta and Yanai (1969) especially in two points; that is, the existence of an upper critical wavelength for instability and the appearance of the unstable waves with $c_{r}<-U_{\max }$. If they had investigated the properties of the waves in the vicinity of $U^{*}=2 / \pi^{2}$ in detail, they would have obtained the same results as those above. In these problems the following points may be important. According to the present study the value of $U^{*}=2 / \pi^{2}$ is a critical value at which any Rossby-Haurwitz wave with $L^{*}<4 / \sqrt{3}$ disappears, but this is not true for $L^{*}>4 / \sqrt{3}$. This can be seen from Fig. 2c. A comparison of Fig. 2d with Fig. $2 \mathrm{c}$ indicates that this value is a critical 
Table 1. Values of $c_{r}{ }^{*}$ of two neutral waves as functions of the number of grids for $U^{*}=0.23$ and $L^{*}=8$.

\begin{tabular}{r|c|c}
\hline $2 \mathrm{~N}$ & Rossby wave & $\begin{array}{c}\text { another kind of } \\
\text { neutral wave }\end{array}$ \\
\hline 10 & -1.194091 & -1.020469 \\
20 & -1.182356 & -1.019616 \\
40 & -1.179382 & -1.020483 \\
60 & -1.178832 & -1.020722 \\
80 & -1.178640 & -1.020807 \\
100 & -1.178551 & -1.020845 \\
\hline
\end{tabular}

one at which another kind of a neutral wave appears for $L^{*}>4 / \sqrt{3}$. (Since negative values of $Z$ around $y=0$ are essential to this neutral wave, this wave should not be regarded as Rossby-Haurwitz wave.) However, there remains a question as to whether a wave of this kind is obtained as a solution of the differential equation (2.1). The authors have not succeeded in attempting to clarify this point. In this paper the result obtained by increasing $N$ will be presented. Table 1 shows the values of $c_{r}{ }^{*}$ of RossbyHaurwitz wave and of the other neutral wave for $U^{*}=0.23$ (see Fig. 2f) and $L^{*}=8$. It is noted that the absolute value of $c_{r}{ }^{*}$ of the latter increases with increasing $N$. It is likely that these neutral waves exist as a solution of (2.1). This suggests the existance of an upper critical wavelength for instability. The second question is whether an unstable wave with $c_{r} *<-1$ exists or not. In the case of a weaterly jet Kuo (1949) stated, without proof, that a wave without a steering current was essentially neutral and that a lower critical phase velocity of unstable waves was characterized by a mimimum velocity of a zonal current. For the zonal flow discussed by Lipps (1962), the quantity $Z$ takes zero at two latitudes in each half of the domain. In this case he showed that the phase velocities and wavelengths of unstable waves are bounded by those of the two neutral waves which propagate at different speeds of $U_{k}$. Since $Z$ in Kuo (1949)'s profile takes zero at only one latitude in each half of the domain and there exists a neutral wave which propagates at a mimimum of zonal speeds, Kuo's conclusion as to a lower critical phase velocity of unstable waves is probably true for his profile. In contrast to the case of a westerly jet, a neutral wave which propagates at a minimum velocity of a zonal flow corresponds to a weak solution in the easterly case when $Z$ is not zero at $y=0$. Furthermore, in most cases of easterly jet profiles, $Z$ takes zero at only one latitude in each half of the domain. Therefore, we have no information of the neutral wave which determines an upper critical wavelength of unstable waves and its phase velocity. Although the present study suggests the existence of an unstable wave without a steering current, this problem is left for future investigations.

\section{Effects of lateral boundaries}

When no rigid boundaries are placed at $y= \pm$ $b$, and zonal flow is absent for $|y|>b$, it is expected that the stability properties obtained in the previous section are largely modified. In particular, this may be true for the wave with a phase velocity which is between 0 and $\left(-\beta / k^{2}\right)$. This can be understood from the equation $\psi^{\prime \prime}=$ $\left(k^{2}+\beta / c\right) \psi$ which should be satisfied for $|y|>b$. In the case of a weaterly jet, the phase velocity of the unstable wave is positive. Therefore, wave properties are not much affected by rigid walls placed at $y= \pm b$, as shown by Kuo (1949) and Yanai and Nitta (1968).

It is difficult to obtain a whole picture of the stability properties in the case of infinite domain. Therefore, we shall consider the case where rigid walls are placed at $y= \pm b_{0}\left(b_{0} \geqq b\right)$. We shall define

$$
\delta \equiv\left(b_{0}-b\right) / b .
$$

Figs. $4 \mathrm{a}$ and $4 \mathrm{~b}$ show how the properties of unstable waves obtained for $\delta=0$ change with $\delta$. The ordinate is $c_{r}^{*}$. The thick solid line indicate unstable waves. The black circles indicate the upper critical wavelength of unstable waves, which corresponds to neutral weak solutions. The chain line represents a relation $c_{r}^{*}=-L^{* 2} / 4 \pi^{2} U^{*}$, which corresponds to $c_{r}=-\beta / k^{2}$. The value of $U^{*}$ is taken to be 0.45 in Fig. $4 a$ and 3.00 in Fig. $4 b$. The number of sub-divisions in the main belt $(|y| \leq b)$ is taken to be 10 in these calculations. It is seen from these figures that longer unstable waves with $-\beta / k^{2}<c_{r}<0$ for $\delta=0$ is largely modified by the increase in $\delta$. Either their phase velocities become less than $\left(-\beta / k^{2}\right)$ or the waves become neutral, although $\left(-\beta / k^{2}\right)$ is not a precise measure for the present discussion.

When $U^{*}$ is not very large $\left(U^{*}<1\right)$, the properties of unstable waves obtained for $\delta=2$ are 


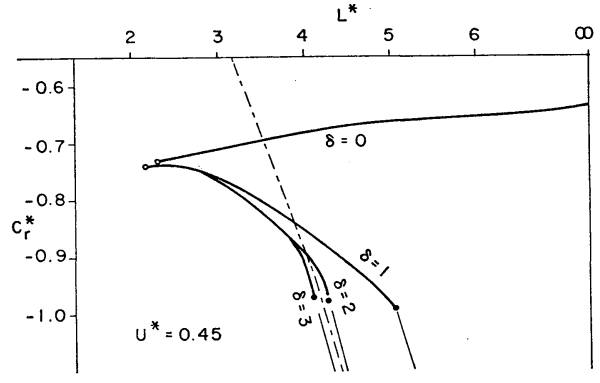

Fig. 4a. Phase velocities of unstable waves (thick solid lines) and Rossby-Haurwitz waves (thin solid lines) as functions of $L^{*}$ for various values of $\delta$. The value of $U^{*}$ is taken to be 0.45 . The chain line indicates the line of $c_{r}=-$ $\beta / k^{2}$.

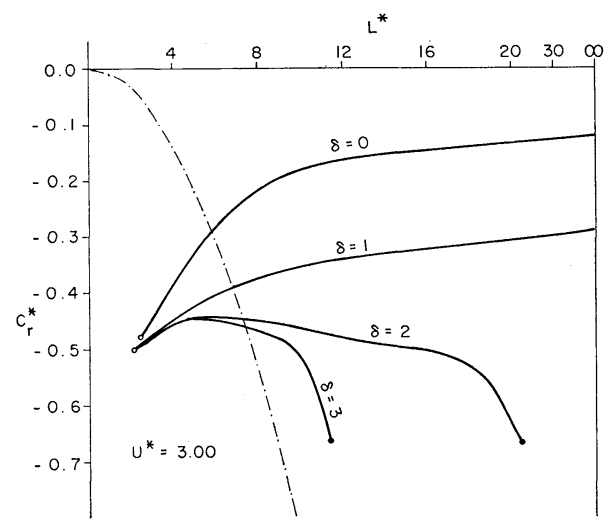

Fig. 4b. Same as Fig. 4a except for $U^{*}=3.0$.

almost the same as those for $\delta=3$. Fig. $4 \mathrm{a}$ is an example. This suggests that the properties of unstable waves in the case of infinite domain are, to some extent, described by a model with $\delta=2$. When $U^{*}$ is 3.0 , however, even $\delta=3$ appears to be unsatisfactory for longer waves, as shown in Fig. 4b. Although the properties of longer waves are greatly dependent upon $\delta$ for large values of $U^{*}, \beta=0$ is a special case. It can be inferred that wave properties for this case scarcely depends upon $\delta$.

When we are interested in the preferred wave only, the value of $\delta$ required to obtain the growth rate and structure to a satisfactory extent decreases with increasing $U^{*}$. This result will be understood in section 5 .

The condition for the existence of unstable waves and their growth rate obtained for $\delta=2$

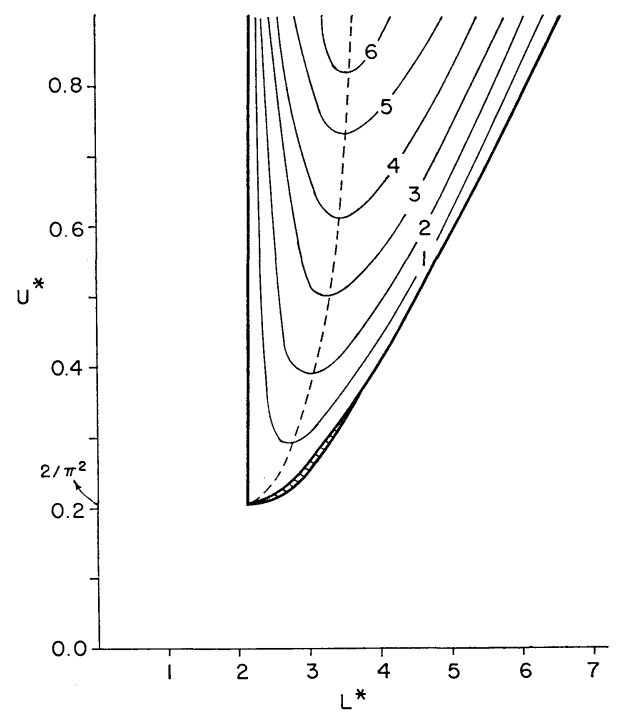

Fig. 5. Growth rates (unit: $\delta b / 20$ ) for $\delta=2$.

are shown in Fig. 5. A comparison with Fig. 3 indicates that the preferred wavelength for $\delta=2$ is much smaller than that for $\delta=0$. For example, when $U^{*}$ is 0.3 , the preferred wavelength is $4.6 \mathrm{~b}$ for $\delta=0$ and $2.8 \mathrm{~b}$ for $\delta=2$. It should be also remarked that, when $U^{*}$ is smaller than 0.5 , the preferred wavelength for $\delta=0$ is outside the unstable regime for $\delta=2$.

The regime for the occurrence of unstable waves with $c_{r}<-U_{\max }$ is shown by the hatched area in Fig. 5. It is found that the existence of such a wave can be ignored for large values of $\delta$.

\section{Other unstable modes}

In the previous section, our concern was directed to the problem of how the properties of unstable waves obtained for $\delta=0$ are dependent upon $\delta$. In the first part of this section we shall discuss another kind of unstable waves which exist only for $\delta>1 / \sqrt{2}$. At first, we shall show the phase velocity diagram obtained for $\delta=2$. Fig. $6 \mathrm{a}$ is a result for $U^{*}=0.28$ and Fig. $6 \mathrm{~b}$ is for $U^{*}=0.45$. The thick solid line indicates an unstable wave. In these examples we have two unstable waves. The shorter wave is the wave that was discussed in section 3 . The longer wave is not obtained for $\delta=0$. The lower critical wavelength of each unstable wave is found to be characterized by the wavelength of a neutral wave with $c_{r}=U_{k}$ (white circle).

In the following, we shall determine the wavelength of the neutral wave in terms of $\delta$ and $U^{*}$. 


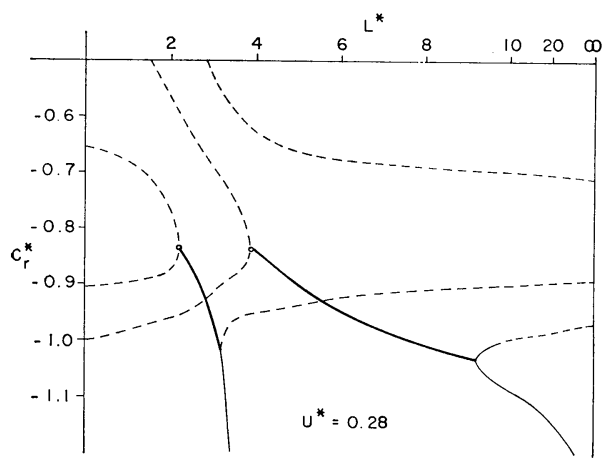

Fig. 6a. Phase velocity diagram for $U^{*}=$ 0.28 and $\delta=2$.



Fig. 6b. Same as Fig. 6a except for $U^{*}=$ 0.45 .

Since its phase velocity is given by (2.4), the equation which should be satisfied by this neutral wave is written as follows:

$$
\frac{d^{2} \psi}{d y^{* 2}}=\left\{\begin{array}{l}
\pi^{2}\left(\frac{4}{L^{* 2}}-1\right) \psi \quad \text { for }\left|y^{*}\right| \leq 1 \\
4 \pi^{2}\left(\frac{1}{L^{* 2}}-\frac{1}{L_{0} * 2}\right) \psi \\
\text { for } 1 \leq\left|y^{*}\right| \leq 1+\delta
\end{array}\right.
$$

where $y^{*}=y / b$ and

$$
L_{0} *=\sqrt{2 \pi^{2} U^{*}+4}
$$

For $L^{*}>L_{0} *$, the symmetric solution of equation (4.1) which satisfies a boundary condition $\phi=0$ at $y^{*}=1+\delta$ is

$$
\psi= \begin{cases}A_{1} \cos \mathrm{my}^{*} & \left(0 \leqq y^{*} \leqq 1\right) \\ B_{1} \sin n\left(y^{*}-1-\delta\right) & \left(1 \leqq y^{*} \leqq 1+\delta\right)\end{cases}
$$

where

$$
\begin{aligned}
& m=\pi \sqrt{1-4 / L * 2} \\
& n=2 \pi \sqrt{1} / L_{0}^{* 2}-1 / L^{* 2} .
\end{aligned}
$$

The condition that $\phi$ and $d \psi / d y^{*}$ should be continuous at $y^{*}=1$ gives the following relation:

$$
m \tan m=n / \tan (n \delta)
$$

For $2<L^{*}<L_{0} *$, the solution for the outer belt is replaced by

$$
\phi=B_{2}\left\{e^{-\bar{n} y *}-e^{-2 \bar{n}(1+\delta)} e^{\bar{n} y *}\right\}
$$

where $\bar{n}=\sqrt{-n^{2}}$. In this case the equation corresponding to $(4.2 \mathrm{a})$ is

$$
m \tan m=\bar{n}\left(1+e^{-2 \overline{n \delta}}\right) /\left(1-e^{-\overline{2 n} \delta}\right)
$$

For $L *<2$, we obtain

$$
-\frac{\bar{m}\left(e^{\bar{m}}-e^{-\bar{m}}\right)}{\left(e^{-m}+e^{-\bar{m}}\right)}=\frac{\bar{n}\left(1+e^{-2 \bar{n} \delta}\right)}{\left(1-e^{-2 \bar{n} \delta}\right)}
$$

where $\bar{m}=\sqrt{-m^{2}}$. This equation (4.2c) can not be satisfied for any values of parameters. Equations (4.2a) and (4.2b) can be easily solved for $\delta$ when values of $U^{*}$ and $L^{*}$ are given.

Fig. 7 shows the wavelength of the neutral wave as functions of $U^{*}$ for various values of $\delta$. The neutral wave in the left part of the chain line $\left(L^{*}<L_{0}^{*}\right)$ is the one that is relevant to the unstable wave discussed in sections 2 and 3 . This wavelength is not dependent upon the value of $\delta$ very much. The wavelength for $\delta=1$ is almost the same as that for infinite domain. On the contrary, the properties of neutral waves obtained for $L^{*}>L_{0} *$ are largely dependent upon $\delta$. This result can be understood from the property of the solution which is sinusoidal in the outer belt. The number of neutral waves increases with increasing $\delta$. This kind of neutral waves do not exist for $\delta<1 / \sqrt{2}$.

It is suggested from the above results that the properties of the longer unstable wave shown in Figs. $6 \mathrm{a}$ and $6 \mathrm{~b}$ are quite dependent upon $\delta$ even if $\delta$ is very large. The number of such unstable waves increases with increasing $\delta$. It is obvious that these waves are different in the meridional mode. The wave discussed in section 3 is the lowest mode. The growth rate decreases with increasiing meridional mode. 


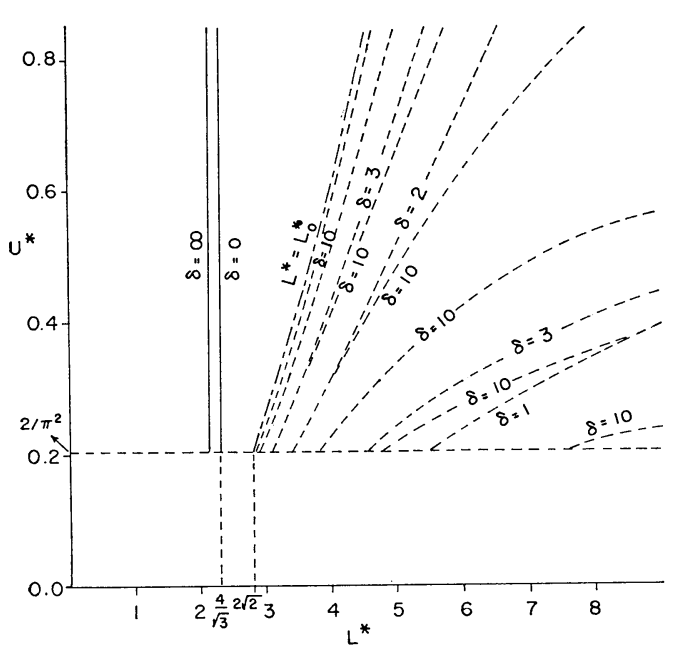

Fig. 7. Values of $L^{*}$ of neutral waves with $c_{r}=U_{\kappa}$ as functions of $U^{*}$ for various values of $\delta$. The solid line indicates a neutral wave obtained as a solution of eq. (4.2b). The dashed lines indicate neutral waves obtained from eq. (4. 2a). In the regime left to the chain line $\left(L^{*}=L_{0}{ }^{*}\right)$, the phase velocity of neutral waves is smaller than $-\beta / k^{2}$ (positive eastward), and in the right regime it is larger than $-\beta / k^{2}$.

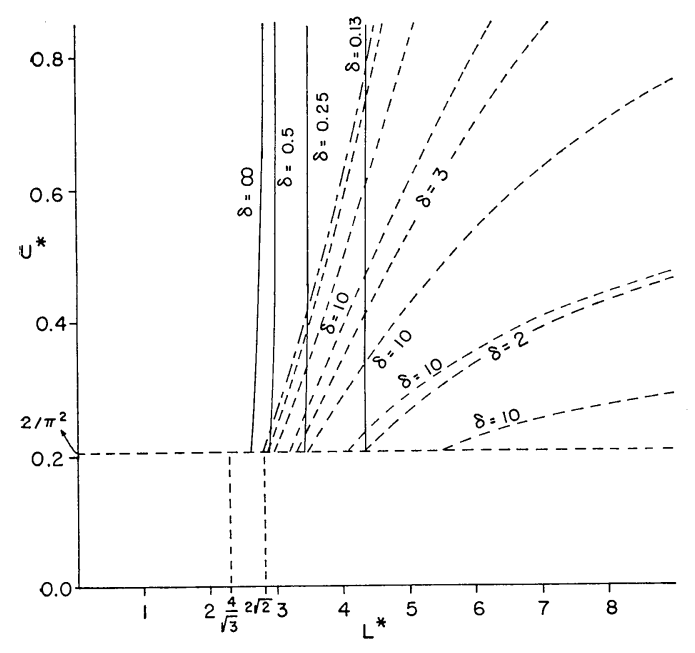

Fig. 8. Same as Fig. 7 except for the antisymmetric mode obtained from (4. 3).

We have discussed symmetric waves in which

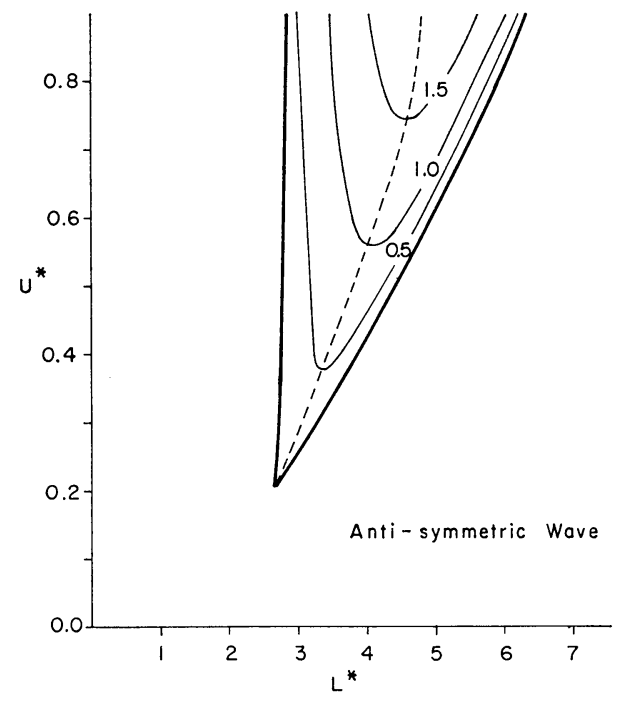

Fig. 9. Growth rates (unit: $\beta b / 20$ ) of the antisymmetric waves as a function of $U^{*}$ and $L^{*}$ for $\delta$ $=2$.

$\phi$ is symmetric with respect to $y=0$. In the following, we shall consider antisymmetric waves. The wavelength of antisymmetric neutral waves with $c=U_{k}$ is determined from the following equation:

$$
-m / \tan m=\left\{\begin{array}{lc}
n / \tan (n \delta) & \left(L_{0} *<L^{*}\right) \\
\bar{n}\left(1+e^{-\overline{2 n} \delta}\right) /\left(1-e^{-2 \bar{n} \delta}\right) & \left(2<L^{*}<L_{0} *\right)
\end{array}\right.
$$

Fig. 8 shows the wavelength as functions of $U^{*}$ for various values of $\delta$. It is found that, when $\delta$ is larger than about 0.5 , there exists a neutral wave whose wavelength scarcely depends upon $\delta$. The wavelength for $\delta=1$ is almost identical to that obtained for infinite domain. As $\delta$ becomes zero, the wavelength of this neutral wave tends to infinity, indicating that no unstable antisymmetric waves exist for $\delta=0$ (Nitta nd Yanai, 1969). In addition to this type of neutral waves, there exist neutral waves whose properties are quite dependent upon $\delta$, as in the case of symmetric waves.

The phase velocity diagram for the antisymmetric case with $U^{*}=0.45$ and $\delta=2$ is similar to that for the symmetric case (Fig. 6b) so that it is not illustrated. The condition for the occurrence of antisymmetric lowest mode and its growth rate are shown in Fig. 9. A comparison with Fig. 5 
indicates that the growth rate of the most preferred wave of the antisymmetric wave is about one-third of that of the symmetric wave.

In contrast to the symmetric mode, an antisymmetric neutral wave with $c=-U_{\max }$ may be obtained as a regular solution of equation (2.1). It remains to be studied whether an upper critical wavelength of antisymmetric unstable waves is bounded by the wavelength of this neutral wave.

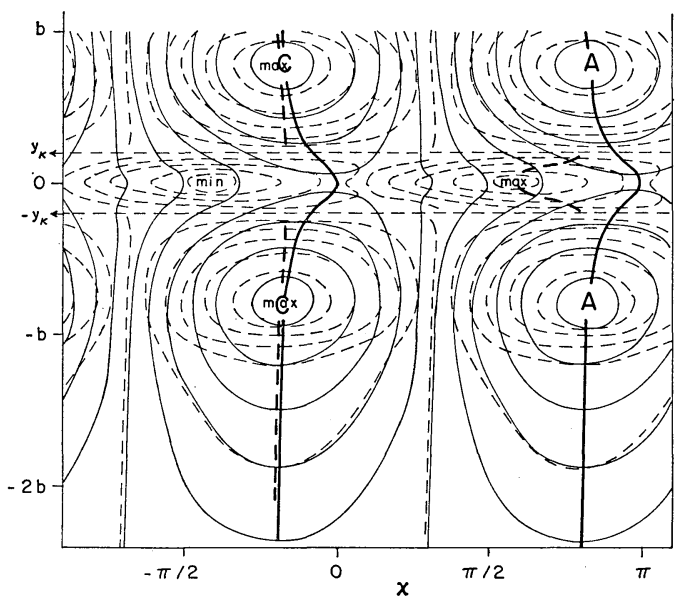

Fig. 10a. Horizontal structure of an unstable wave (lowest meridional mode) of $L^{*}=$ 2.9 for $U^{*}=0.25$ and $\delta=2$. The fields are symmetric with respect to $y=0$. For details see text.

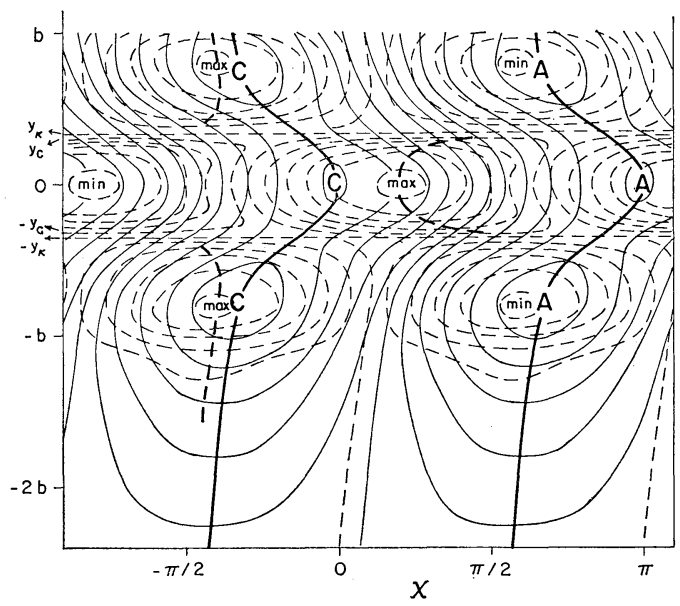

Fig. 10b. Same as Fig. 10a except for $L^{*}$ $=3.2$ and $U^{*}=0.45$.

\section{Structure of the unstable wave}

The unstable wave of the type discussed in section 3 is most interesting among various types of unstable waves. We shall confine our discussions of the structure to this unstable wave. Three typical structures in the horizontal plane are shown in Figs. 10a, 10b and 10c. The value of $U^{*}$ is taken to be 0.25 in Fig. $10 \mathrm{a}, 0.45$ in Fig. $10 \mathrm{~b}$ and 2.0 in Fig. 10c. The most preferred wave is chosen in each case. The phase velocity is as follows: $c_{r}<-U_{\max }$ in Fig. 10a, $-U_{\max }<$ $c_{r}<U_{k}$ in Fig. $10 \mathrm{~b}$ and $U_{k}<c_{r}$ in Fig. 10c. $y_{k}$ is the latitude where $Z$ is zero and $y_{c}$ is the latitude where $c_{r}=U$. The value of $\delta$ used for these figures is 2 . These figures do not cover the entire domain for the save of space. The streamfunction field is drawn by the solid lines. The centers of cyclonic and anticyclonic circulations are indicated by $\mathrm{C}$ and $\mathrm{A}$, respectively. The thick solid lines indicate streamfunction trough and ridge lines. The relative vorticity field is drawn by the dashed lines. The locations of extrema of the relative vorticity are indicated by max and min. The phase lines of the maximum vorticity are indicated by the thick dashed lines.

As a matter of fact, the trough line of the barotropic unstable wave tilts eastward with $i_{\text {ncreasing }} y$ in a region of negative $y$ where $\partial U /$ $\partial y$ is negative and tilts westward in a region of positive $y$ where $\partial U / \partial y$ is positive. Another notable feature is the appearance of circulation centers located at $y \fallingdotseq \pm 0.8 \mathrm{~b}$ in Figs. $10 \mathrm{a}$ and



Fig. 10c. Same as Fig. 10a except for $L^{*}=$ 3.7 and $U^{*}=2.0$. 
10b. A circulation center is not found at $y=0$ in Fig. 10a. This is interpreted as follows. As is clear from equation (2.1), the change of vorticity is caused by meridional transport of the vorticity of the basic flow and by advection due to basic flow. In the central belt $\left(|y|<y_{k}\right)$ where $Z$ is negative, the relative vorticity and southerly wind should be positively correlated in the unstable wave because southerly wind act to increase the vorticity. In the case of Fig. 10a, wave speed is faster than the easterly current. Therefore, the phase line of the maximum vorticity should be located to the east of the maximum southerly wind in a region of negative $Z$. These require that the maximum vorticity in the central belt be located less than $1 / 4$ cycle to the west of the ridge. This means that the vorticity should be negative around the trough in the central belt. Therefore, the amplitude of the streamfunction takes a minimum at $y=0$ in Fig. 10a.

In the case where $-U_{\max }<c_{r}<U_{k}$, the maximum vorticity in the central belt is located just to the east of the trough because the easterly current is faster than the wave speed in a region $|y|<y_{c}$ and $Z$ is negative. This means that the vorticity around the trough in the central belt is poitive. In the case of Fig. $10 \mathrm{~b}$ a weak circulation center is found at $y=0$. The amplitude of the streamfunction at $y=0$ relative to the amplitude around $\pm 0.8 \mathrm{~b}$ increases with increasing $U^{*}$. When $U^{*}$ exceeds a certain value, the extrema of the streamfunction around $\pm 0.8 \mathrm{~b}$ are not noticeable, as seen in Fig. 10c.

In addition to an extremum of vorticity located at $y=0$, there are two extrema of vorticity around $\pm 0.8 \mathrm{~b}$. The intensity of this extremum is dependent upon the value of $Z$ around this latitude. This location is slightly west of the trough because $Z$ is positive and $\left(c_{r}-U\right)$ is negative around this latitude. As is obvious from (2.1), the vorticity vanishes at $y_{k}$ where $Z$ is zero. The phase line of vorticity jumps at this latitude. This can be also understood from the sign of $Z$ and phase velocity relative to the zonal current.

A comparison of these three figures indicate that the amplitude of the streamfunction in the outer belts, for instance, at $y= \pm 2 b$ in the preferred wave decreases with increasing $U^{*}$. This result is related to the validity of imposed boundary conditions discussed in section 3 .

\section{Concluding remarks}

The properties of the barotropic unstable wave in an easterly zonal current with a sine-profile jet have been discussed by solving finite difference equations analogous to the barotropic vorticity equation on a $\beta$-plane. It was found that the stability properties of an easterly jet are different from those of a westerly jet in several respects. In the case of an easterly jet, there exist a number of unstable waves with different meridional modes. The lowest and second meridional modes appear to be well described by a $\beta$-plane model, whereas higher modes are not properly described because the properties of higher modes are quite dependent upon the position of lateral boundaries. It can be expected that a spherical coordinate model gives appropriate description. Although the relative importance of all unstable waves cannot be determined until we discuss the problem by the use of a spherical coordinate model, it is probable that the lowest mode has the largest growth rate and therefore it is most interesting.

The horizontal structure of the lowest mode was discussed in section 5. Three typical structures are found in accordance with the phase velocity. In view of the growth rate, the structures illustrated in Figs. 10b and 10c are interesting. The structure in the case of easterly jet is different from that in the case of westerly jet with respect to the meridional profile of the wave amplitude, horizontal tilt of the wave axis, the phase relation between the streamfunction trough and vorticity and so on. In order to describe the properties of the unstable wave appropriately, it is required that lateral boundaries be placed at a sufficient distance from the belt of an easterly current. The minimum distance which can be accepted decreases with increasing horizontal shear and with decreasing wavelength.

The present study also suggests that there exists an unstable wave which propagates faster than a maximum easterly current. This wave is, however, practically unimportant, even if it exists theoretically, because the growth rate is very small. Furthermore, in the actual atmosphere where vertical shear of a zonal current exists, it is not likely that unstable waves of this type are found.

As mentioned in section 1, this study was started as a preliminary one to explain the easterly wave in the tropical troposphere. As a next step of 
this study, the authors have investigated the three-dimensional structure of the barotropic unstable wave by the use of a quasi-geostrophic model and compared the structures with observed ones. The vorticity field illustrated in Fig. 10 helps to understand the vertical motion field obtained in the three-dimensional model. Results will be reported in another paper (Yamasaki and Wada, 1972).

\section{Acknowledgments}

The authors wish to express their thanks to Prof. K. Ooyama and Prof. M. Yanai for their comments on this work.

\section{References}

Brown, J.A., 1969: A numerical investigation of hydrodynamic instability and energy conversions in the quasi-geostrophic atmosphere: Part I. J. Atmos. Sci., 26, 352-365.

Chang, C.P., 1970: Westward propagating cloud patterns in the tropical Pacific as seen from timecomposite satellite photographs. J. Atmos. Sci.,' 27, 133-138.

, V.F. Morris and J.M. Wallace, 1970: A statistical study of easterly waves in the western Pacific: July-December 1964. J. Atmos. Sci., 27, 195-201.

Charney, J.G., 1969: A further note on large-scale motions in the tropics. J. Atmos. Sci., 26, 182185.

Eliasen, E., 1954: Numerical solutions of the perturbation equation for linear flow. Tellus, 6, 183-191.

Haltiner, G.J., and R.T. Song, 1962: Dynamic instability in barotropic flow. Tellus, 14, 383-393.

Hayashi, Y., 1970: A theory of large-scale equatorial waves generated by condensation heat and accerelating the zonal wind. J. Meteor. Soc. Japan, 48, 140-160.

Krishnamurti, T.N., 1969: An experiment in numerical prediction in equatorial latitudes. Quart. J. Roy. Meteor. Soc., 95, 594-620.

Kuo, H.L., 1949: Dynamic instability of two-dimensional nondivergent flow in a barotropic atmosphere. J. Meteor., 6, 105-122.

Lipps, F.B., 1962: The barotropic stability of the mean winds in the atmosphere. J. Fluid Mech., 12, 397-407.

, 1965: The stability of an asymmetric zonal current in the atmosphere. J. Fluid Mech., 21, 225-239. disturbances. Mon. Wea. Rev., 98, 122-131.

Mak, M.K., 1969: Laterally driven stochastic motions in the tropics. J. Atmos. Sci., 26, 41-64.

Manabe, S., and J. Smagorinsky, 1967: Simulated climatology of a general circulation model with a hydrologic cycle II. Analysis of the tropical atmosphere. Mon. Wea. Rev., 95, 155-169.

Manabe, S., J.L. Holloway, and H.M. Stone, 1970: Tropical circulation in a time-integration of a global model of the atmosphere. J. Atmos. Sci., 27, 580-613.

Nitta, Tsuyoshi, 1970 a: On the role of transient eddies in the tropical troposphere. J. Meteor. Soc. Japan, 48, 348-359.

- $1970 \mathrm{~b}$ : A study of generation and conversion of eddy available potential energy in the tropics. J. Meteor. Soc. Japan, 48, 524-528.

, and M. Yanai, 1969: A note on the barotropic instability of the tropical easterly current. J. Meteor. Soc. Japan, 47, 127-130.

Ooyama, K., 1971: On the stability of wave disturbances in the tropical easterlies. (to be submitted)

Palmer, C.E., 1952: Review of tropical meteorology. Quart. J. Roy. Meteor. Soc., 78, 126-163.

Riehl, H., 1954: Tropical meteorology. McGraw Hill, $392 \mathrm{p}$.

Wallace, J.M., and C.P. Chang, 1969: Spectrum analysis of large-scale wave disturbances in the tropical lower troposphere. J. Atmos. Sci., 26, 1010-1025.

Wiin-Nielsen, A., 1961: On short- and long-term variations in quasi-barotropic flow. Mon. Wea. Rev., 89, 461-476.

Yamasaki, M., 1969: Large-scale disturbances in the conditionally unstable atmosphere in low latitudes. Pap. Meteor. Geophys., 20, 289-336. , 1971: A further study of wave disturbances in the conditionally unstable model tropics. J. Meteor. Soc. Japan, 49, , and M. Wada, 1972: Vertical structure of the barotropic unstable wave in a tropical easterly current. J. Meteor. Soc. Japan, (to be submitted).

Yanai, M., 1961: Dynamical aspects of typhoon formation. J. Meteor. Soc. Japan, 39, 282-309.

, and Ts. Nitta, 1968: Finite difference approximations for the barotropic instability problem. J. Meteor. Soc. Japan, 46, 389-403.

- T. Maruyama, Ts. Nitta, and Y. Hayashi, 1968: Power spectra of large-scale disturbances over the tropical Pacific. J. Meteor. Soc. Japan, 46, 308-323. 


\title{
東風帯状流の順圧不安定
}

\author{
山岬 正紀 - 和 田 美 鈴

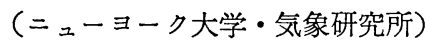

新田・柳井（1969）の発展として, 順圧東風帯状流中の非発散な順圧不安定波の性質をより詳しく調べた. Kuo （1949）等によって論じられた西風帯状流の順圧不安定と比べて東風帯状流の順圧不安定は多くの異なった特徵をも っていることがわかった．たとえば，一般流より速く西進する順圧不安定波の存在の可能性，種々の南北モードをも った多くの不安定波の存在, 南北の境界に対する波の性質の強い依存性, 波の振幅の緯度分布の特異な振舞い等があ


定波をもらまく記述するためには球座標を用いる必要がある。 